\title{
Nanoscale
}

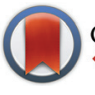

CrossMark \&click for updates

Cite this: Nanoscale, 2015, 7, 5654

\section{Quantitatively probing propensity for structural transitions in engineered virus nanoparticles by single-molecule mechanical analysis $\uparrow$}

\begin{abstract}
Milagros Castellanos, Pablo J. P. Carrillo and Mauricio G. Mateu*
Viruses are increasingly being studied from the perspective of fundamental physics at the nanoscale as biologically evolved nanodevices with many technological applications. In viral particles of the minute virus of mice (MVM), folded segments of the single-stranded DNA genome are bound to the capsid inner wall and act as molecular buttresses that increase locally the mechanical stiffness of the particle. We have explored whether a quantitative linkage exists in MVM particles between their DNA-mediated stiffening and impairment of a heat-induced, virus-inactivating structural change. A series of structurally modified virus particles with disrupted capsid-DNA interactions and/or distorted capsid cavities close to the DNAbinding sites were engineered and characterized, both in classic kinetics assays and by single-molecule mechanical analysis using atomic force microscopy. The rate constant of the virus inactivation reaction was found to decrease exponentially with the increase in elastic constant (stiffness) of the regions closer to DNA-binding sites. The application of transition state theory suggests that the height of the free energy barrier of the virus-inactivating structural transition increases linearly with local mechanical stiffness. From a virological perspective, the results indicate that infectious MVM particles may have acquired the biological advantage of increased survival under thermal stress by evolving architectural elements that rigidify the particle and impair non-productive structural changes. From a nanotechnological perspective, this study provides proof of principle that determination of mechanical stiffness and its manipulation by protein engineering may be applied for quantitatively probing and tuning the conformational dynamics of virus-based and other protein-based nanoassemblies.
\end{abstract}

Received 28th November 2014 Accepted 15th February 2015

DOI: $10.1039 / c 4 n r 07046 a$

www.rsc.org/nanoscale able as nanodevices or nanocontainers for different nano/biotechnological applications. ${ }^{1-7}$

The mechanical properties of viruses and their protein capsids are being experimentally probed in single-particle experiments using atomic force microscopy (AFM) in a liquid, as established in a trend-setting work on bacteriophage $\$ 29$ mechanics. ${ }^{12}$ To determine stiffness, single virus particles under close to physiological conditions are subjected to a mechanical force, and the elastic constant $k_{\mathrm{e}}$ of the indented region along the direction of the applied force is determined. ${ }^{18}$ Theoretical approaches are also being used to investigate stiffness and other mechanical properties of idealized virus models. ${ }^{9,19}$ Both experiment and theory have already provided important insights into virus mechanics and its biological implications. ${ }^{9,12-17,19,20}$

Despite these advances, both fundamental and nanotechnologically relevant questions on the mechanical features of virus particles and its relationship to virus structure and function remain essentially unanswered. ${ }^{14}$ One aspect that needs to be fully addressed is the connection between mechanical elasticity and conformational dynamics in viral nanoparticles and other protein-based bioassemblies. de Madrid, Madrid 28049, Spain. E-mail: mgarcia@cbm.csic.es

$\dagger$ Electronic supplementary information (ESI) available. See DOI: 10.1039/ c4nr07046a

$\$$ Present address: Centro Nacional de Biotecnología (CSIC), Campus de Cantoblanco, 28049 Madrid, Spain. 
Viruses are highly dynamic supramolecular complexes. For example, in many virus species newly assembled particles mature through their conformational reorganization into metastable infectious virions. Some metastable virions have acquired through evolution a high physicochemical resistance against extracellular disruption, but they are poised to release their nucleic acid in the host cell through additional rearrangements triggered by specific factors that decrease the free energy barrier of the reaction. ${ }^{21-28}$

Conformational dynamics not only provides virions with a selective advantage; it may also constitute an Achilles' heel by facilitating their inactivation. Heating is one of the most prevalent aggressions virions must endure in their journey between cells and organisms. Heat-induced inactivation of virus infectivity may occur either by disintegration of the virion or, frequently, through a conformational rearrangement without losing overall integrity. ${ }^{21,24,29,30}$ In many instances, the underlying structural change has not been characterized. However, some virions are inactivated because heat in the extracellular environment can untimely trigger a conformational rearrangement that normally occurs under biological control during the infectious cycle. ${ }^{21,24}$ In general, thermal instability and inactivation of virions involve irreversible, kinetically controlled structural changes. ${ }^{31}$

From a nanotechnological perspective, the biologically evolved compromise between conformational stability and instability of virus particles may prove either desirable or undesirable, depending on the intended application. For example, structural rearrangements, even without particle disintegration, may lead to untimely release of the cargo in nanocontainers; distortion of epitopes in nanoparticle-based vaccines; or detrimental changes in functionality or relative position of active moieties in nanosensors or nanomaterials. ${ }^{3}$
Thus, it may be important to develop strategies to chemically and/or genetically tune the propensity of viral nanoparticles and other bioassemblies to undergo conformational transitions, and simple but quantitative analytical methods to test if such a propensity has been effectively modified.

We use the minute virus of mice (MVM), a small (25 nm diameter) and structurally simple parvovirus, ${ }^{32,33}$ as a model to study conformational stability and dynamics of virus particles. During MVM morphogenesis, a single-stranded (ss)DNA genome is encapsidated $^{34}$ within a previously assembled $T=1$ icosahedral capsid made of 60 structurally equivalent protein subunits (Fig. 1). Short segments of the internalized ssDNA are then folded as "wedges" that bind equivalent concave sites at the capsid inner wall ${ }^{32,33}$ (Fig. 1B and D).

These DNA wedges in the MVM virion act as molecular buttresses that increase mechanical stiffness at regions centered around twofold (S2) or threefold (S3) symmetry axes, close to the DNA binding sites (Fig. 1B and C). ${ }^{35,36}$ Regions centered around channels (pores) located at the fivefold (S5) axes are, however, kept free from bound DNA (Fig. 1B and C), and remain as elastic as in the DNA-free (empty) capsid. ${ }^{35,36}$ The S5 regions are involved in conformational transitions $s^{37,38}$ leading to through-pore molecular translocation events required for virus infectivity, ${ }^{39-45}$ and the preserved mechanical flexibility at these regions facilitates these biologically essential conformational transitions. ${ }^{46}$ Also, preliminary observations ${ }^{36,47}$ led us to advance the hypothesis of a connection between DNA-mediated increased mechanical stiffness at S2/S3 regions and impairment of a heat-induced, virus-inactivating structural change. ${ }^{36}$ The above experimental evidence led us to hypothesize that the conflict between beneficial and detrimental effects of conformational dynamics has been met in this virus by evolving an anisotropic mechanical behavior,
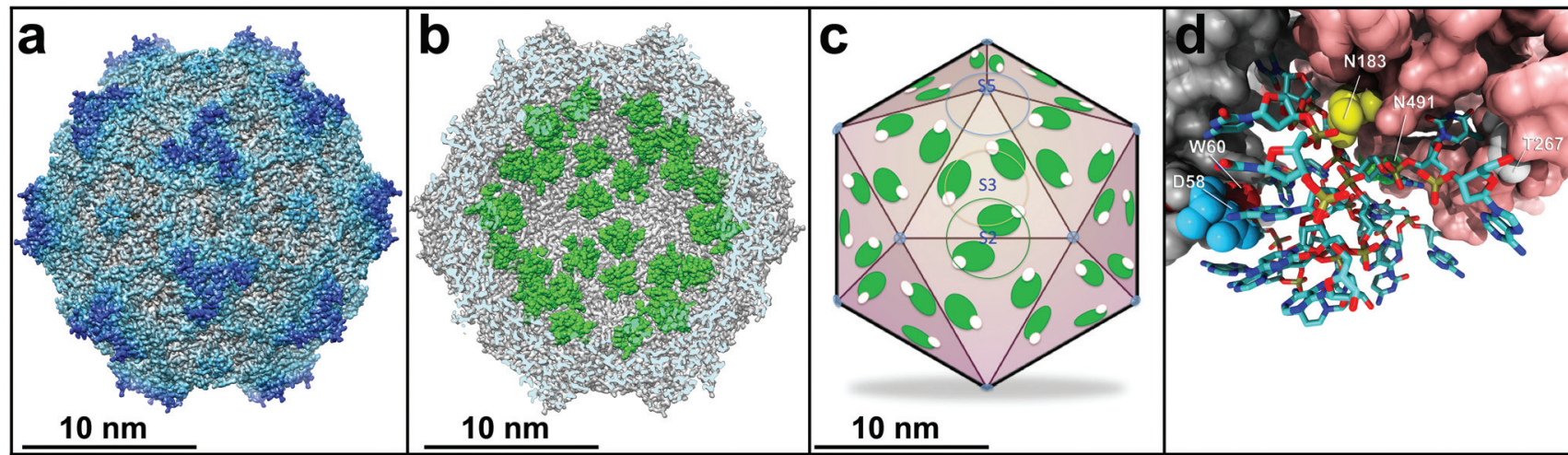

Fig. 1 The MVM virion. (a) Atomic model of the crystal structure of the MVM virion ${ }^{32,33}$ (PDB code 1Z1C). The maximum virion diameter is about $25 \mathrm{~nm}$. (b) Cross-section of the MVM virion structure. DNA segments bound to equivalent sites at the capsid inner wall are colored green. (c) Scheme of the MVM capsid architecture. The 20 equivalent trimers of the capsid protein that constitute the stable building blocks for MVM capsid assembly fit in the 20 triangular faces of the icosahedron. Two-, three- and five-fold icosahedral symmetry axes are respectively labelled S2, S3, and S5. Approximate positions and sizes of equivalent DNA-binding sites and cavities in the virion, projected onto the idealized icosahedral surface, are respectively indicated by green or white ellipses. The capsid pores are indicated as small cyan ellipses at each 55 vertex. To determine the elastic constant at S2, S3 or S5 regions, the virions were indented approximately within the areas indicated by green, yellow or blue open circles, respectively. (d) A close-up view of one of the 60 equivalent DNA-binding sites in the capsid inner wall represented as a space-filling model. The folded DNA segment ("wedge") bound to it is represented in a stick model. Capsid amino acid residues whose interactions with the DNA wedge were removed by individual mutations to alanine are shown in different colors and labelled. 
as a compromise to acquire enough rigidity to impair an inactivating conformational transition, without reducing the local elasticity that mediates another conformational rearrangement required for virus infection. ${ }^{46}$ We also found during those studies that both global and local mechanical elasticity in a viral particle can be manipulated (either increased or decreased) by genetically replacing specific chemical groups and altering some non-covalent intracapsid or capsid-nucleic acid interactions. ${ }^{36,46}$

Based on the above observations, in the present study we have engineered and analyzed a series of structurally modified virus particles to address two questions: (i) is there a linkage between the DNA-mediated increase in mechanical stiffness at the $\mathrm{S} 2 / \mathrm{S} 3$ regions in the MVM virion, and the impairment of a heat-induced, virus-inactivating structural change? (ii) Can mechanical elasticity and conformational dynamics in the MVM virion be quantitatively related? The results indicate that a linear relationship exists between mechanical stiffening of particular regions in the MVM virion and the free energy barrier of a structural transition in which that region is involved.

\section{Results}

\section{Disruption of different capsid-DNA interactions in the MVM virion reduce the local mechanical stiffness to different extents}

The crystal structure of the MVM virion ${ }^{32,33}$ was used to inspect the DNA-binding sites at the capsid inner wall. Individual substitutions by alanine of residues D58, N183, W60, T267 and N491 located at each of these sites (Fig. 1D and Table 1) were chosen for comparative structure-function analysis of virion mechanical elasticity and resistance to thermal inactivation.

Truncation of the side chains of the first three residues by mutation to alanine disrupts relatively strong interactions between the capsid and the DNA wedges (hydrogen bonds for D58, N183 or many hydrophobic contacts for W60, in addition to a number of van der Waals interactions in all three cases). Mutation to alanine of N491 and T267 disrupts weaker interactions (involving van der Waals contacts only). Those five mutations neither disrupt any significant interaction with other capsid residues, nor introduce groups that could establish new interactions; thus, any observed effect could be specifically attributed to removal of capsid-DNA interactions. Most of the residues chosen and their interactions with the DNA are well conserved in two strains of $\mathrm{MVM}^{33}$ and the evolutionarily related canine parvovirus, ${ }^{48}$ which supports their biological relevance.

The five chosen mutant virions and the non-mutated virion were produced through parallel transfections of susceptible cells, and the viral progeny was quantified in normalized titration assays. All mutants except one were nearly as infectious as the non-mutated virus, with relative titers between 0.4 and 1.2; mutant N183A was somewhat less infectious (relative titer 0.15) (Table 1).
Table 1 Effects of mutations in the MVM virion on virus infectivity and thermal inactivation rate constant $k_{\text {in }}$

\begin{tabular}{lll}
\hline Virion & $\begin{array}{l}\text { Relative } \\
\text { infectivity }^{a}\end{array}$ & $\begin{array}{l}\text { Relative inactivation } \\
\text { rate constant } k_{\text {in }}{ }^{b}\end{array}$ \\
\hline $\begin{array}{l}\text { Parent (non-mutated) } \\
\text { Mutated in DNA sites }\end{array}$ & 1.00 & 1.00 \\
D58A & $0.45 \pm 0.07$ & $7.00 \pm 1.96$ \\
W60A & $0.39 \pm 0.19$ & $8.05 \pm 0.91$ \\
N183A & $0.15 \pm 0.01$ & $9.00 \pm 2.54$ \\
T267A & $0.43 \pm 0.09$ & $1.73 \pm 0.25$ \\
N491A & $1.17 \pm 0.05$ & $2.09 \pm 0.83$ \\
D58A/N183A & $0.01 \pm 0.01$ & $3.40 \pm 0.67$ \\
Mutated in cavities & & \\
L113F & $0.88 \pm 0.20$ & $0.90 \pm 0.06$ \\
L143F & $0.99 \pm 0.13$ & $1.03 \pm 0.18$ \\
Mutated in DNA sites and cavities & \\
D58A/L113F & Lethal & Not applicable \\
D58A/L143F & $0.05 \pm 0.02$ & $3.45 \pm 0.32$ \\
N183A/L113F & $0.03 \pm 0.01$ & $4.16 \pm 1.76$ \\
N183A/L143F & $0.08 \pm 0.02$ & $3.85 \pm 1.93$
\end{tabular}

${ }^{a}$ The indicated values are the average of $2-4$ independent experiments. The absolute titer determined for the parent virus in 4 experiments was $(2.15 \pm 1.61) \times 10^{7} \mathrm{pfu} \mathrm{ml}^{-1}$. $^{b}$ The indicated values are the average of 2-3 independent experiments. The absolute inactivation rate constant determined for the parent virion in 14 experiments (average \pm standard deviation) was $(5.4 \pm 1.6) \times 10^{-4} \mathrm{~s}^{-1}$.

Mutant and non-mutated virions were purified using the same conditions, and subjected to mechanical analysis by AFM in single-particle indentation experiments in physiological buffer. ${ }^{35}$ High-resolution AFM imaging served to determine the region of each virus particle that was to be indented (S2, S3 or S5 regions centered around the corresponding symmetry axes) (Fig. 2). ${ }^{35}$ For each mutant and region indented many measurements were carried out and averaged (Table 2).

All five mutant virions yielded $k_{\mathrm{e}}$ values at $\mathrm{S} 5$ regions that were indistinguishable from the corresponding value obtained for the non-mutated virion; the same was observed with any other infectious mutant virion tested in this study (Fig. S1 of ESI $\dagger$ and Table 2). This result was expected from the inextricable linkage we had already found between preservation of mechanical elasticity at the capsid pore (S5) regions, and pres-

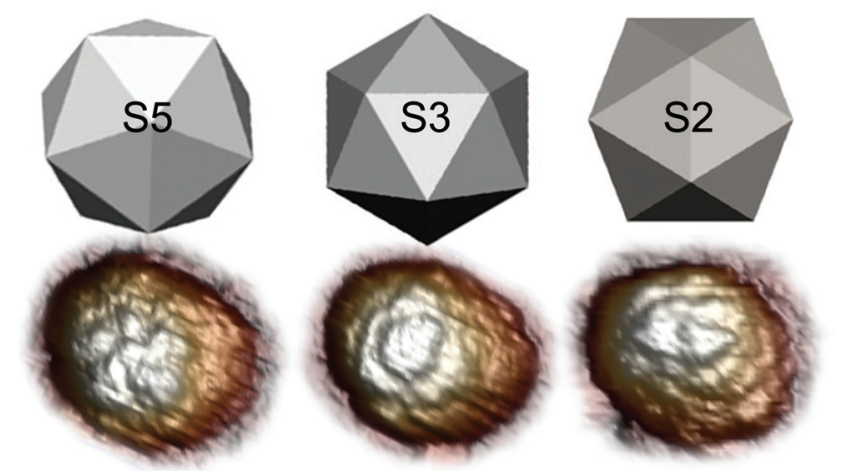

Fig. 2 Icosahedral model and actual AFM images of MVM virions. They are viewed along a five-fold (S5), three-fold (S3) or two-fold (S2) symmetry axis. 
Table 2 Effects of mutations in the MVM virion on the elastic constant $k_{\mathrm{e}}$ at S2, S3 and S5 regions

\begin{tabular}{|c|c|c|c|c|c|c|c|c|c|c|c|c|c|c|c|}
\hline Virion & \multicolumn{5}{|l|}{$\mathrm{S} 2$} & \multicolumn{5}{|l|}{ S3 } & \multicolumn{5}{|l|}{ S5 } \\
\hline D58A & $0.82 \pm 0.10$ & 46 & 5 & 0.02 & $9.9 \times 10^{-13}$ & $0.57 \pm 0.10$ & 66 & 7 & 0.01 & $1.2 \times 10^{-14}$ & $0.55 \pm 0.06$ & 64 & 6 & 0.01 & 0.05 \\
\hline W60A & $0.68 \pm 0.15$ & 52 & 4 & 0.02 & $1.7 \times 10^{-29}$ & $0.70 \pm 0.15$ & 23 & 6 & 0.03 & $1.3 \times 10^{-4}$ & $0.61 \pm 0.19$ & 36 & 4 & 0.03 & 0.44 \\
\hline N183A & $0.74 \pm 0.19$ & 88 & 12 & 0.02 & $2.0 \times 10^{-11}$ & $0.58 \pm 0.15$ & 79 & 15 & 0.02 & $1.2 \times 10^{-11}$ & $0.57 \pm 0.10$ & 34 & 8 & 0.02 & 0.80 \\
\hline T267A & $1.18 \pm 0.26$ & 48 & 8 & 0.04 & 0.17 & $0.81 \pm 0.17$ & 25 & 6 & 0.03 & 0.27 & $0.56 \pm 0.10$ & 48 & 9 & 0.01 & 0.23 \\
\hline L113F & $1.12 \pm 0.25$ & 60 & 9 & 0.03 & 0.003 & $0.89 \pm 0.20$ & 58 & 8 & 0.03 & 0.30 & $0.55 \pm 0.11$ & 64 & 6 & 0.01 & 0.02 \\
\hline L143F & $1.34 \pm 0.26$ & 48 & 6 & 0.04 & 0.07 & $0.88 \pm 0.22$ & 57 & 10 & 0.03 & 0.47 & $0.57 \pm 0.11$ & 49 & 7 & 0.02 & 0.46 \\
\hline D58A/L143F & $1.11 \pm 0.25$ & 42 & 8 & 0.04 & 0.004 & $0.77 \pm 0.16$ & 37 & 8 & 0.03 & 0.01 & $0.57 \pm 0.11$ & 63 & 7 & 0.01 & 0.27 \\
\hline N183A/L113F & $1.03 \pm 0.23$ & 60 & 5 & 0.03 & $9.4 \times 10^{-7}$ & $0.78 \pm 0.18$ & 61 & 4 & 0.02 & 0.01 & $0.58 \pm 0.12$ & 17 & 2 & 0.03 & 0.79 \\
\hline N183A/L143F & $1.03 \pm 0.26$ & 50 & 7 & 0.04 & $1.5 \times 10^{-5}$ & $0.81 \pm 0.19$ & 27 & 4 & 0.04 & 0.26 & $0.61 \pm 0.11$ & 43 & 6 & 0.02 & 0.34 \\
\hline
\end{tabular}

${ }^{a}$ Average elastic constant $k_{\mathrm{e}} \pm$ standard deviation. ${ }^{b}$ Number of measurements (Fz curves) and virions (n) used. ${ }^{c}$ Standard error of the $k_{\mathrm{e}}$ mean.

${ }^{d}$ Value of $T$ in a Student's $t$-test (see text).

ervation of conformational dynamics at those regions as required for virus infectivity. ${ }^{46}$

In contrast, the five mutations had different effects on the mechanical stiffness of the virion at S2 regions (Fig. 3 and Table 2) and S3 regions (Fig. S2 of ESI $\dagger$ and Table 2) that include the DNA-binding sites (Fig. 1C). Mutations D58A, N183A and W60A, which disrupt more energetic capsid-DNA interactions, caused larger reductions in $k_{\mathrm{e}}$ values, approaching the relatively low $k_{\mathrm{e}}$ values of the DNA-free MVM capsid, although the full DNA was still inside the mutant virions; mutations N491A and T267A, which disrupt weaker capsidDNA interactions, respectively, reduced only the stiffness of the (more rigid) S2 regions or had an insignificant effect on virion stiffness (Table 2).
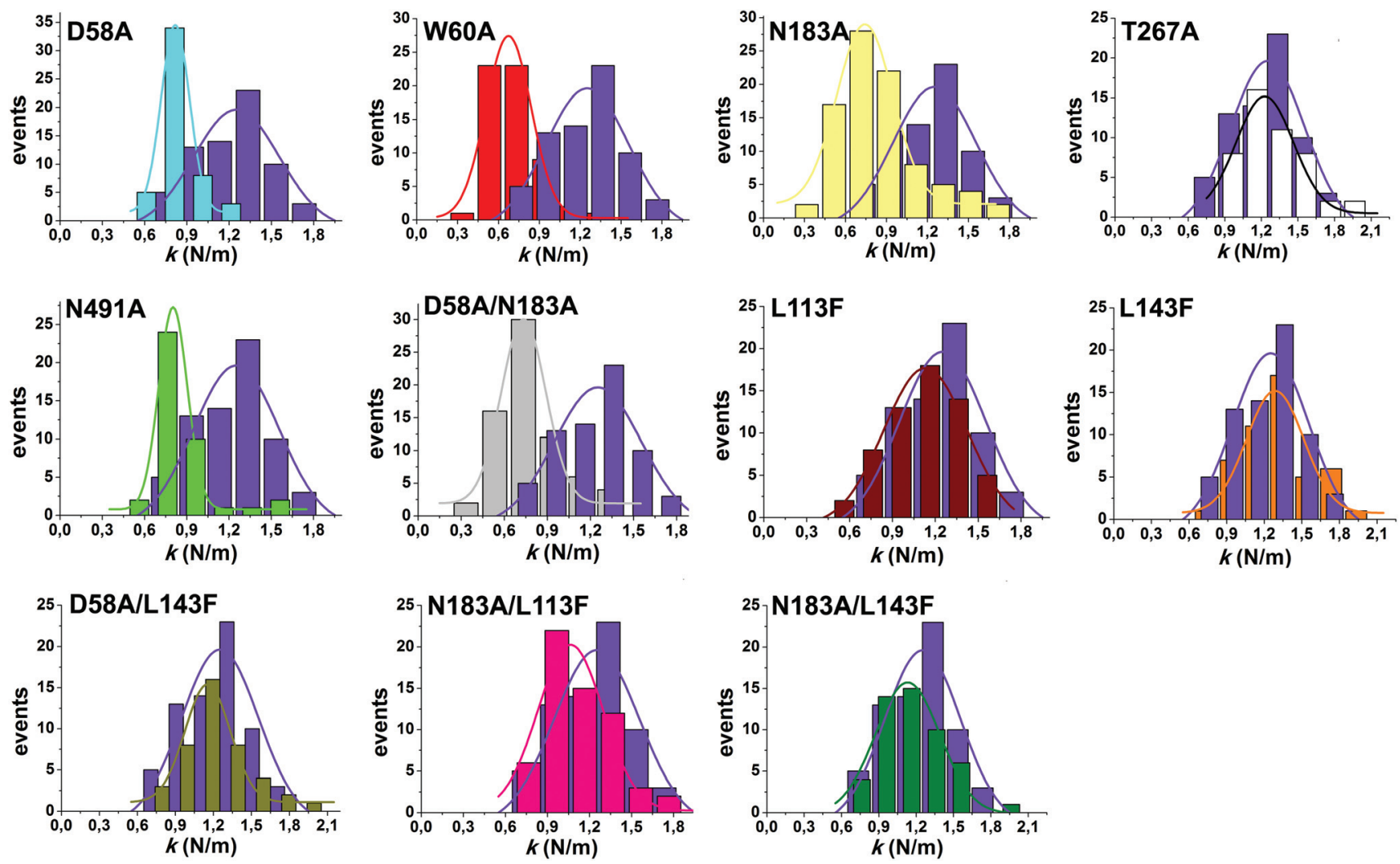

Fig. 3 Comparison of the mechanical stiffness at S2 regions between the non-mutated and mutant MVM virions. Each histogram depicts the number of determinations relative to the $k_{\mathrm{e}}$ obtained for a mutant virion. In each graph, the histogram in purple corresponds to the parent virion, and the one in a different color to a mutant virion, identified by a label (upper left corner). For complete data and statistical analysis see Table 2. 
Reductions in local mechanical stiffness by removing multiple capsid-DNA interactions are non-additive

Disruption of certain capsid-DNA interactions made the S2/S3 regions in the virion almost as flexible as in the empty capsid. We next tested whether stiffness could be further reduced by disrupting further capsid-DNA interactions, through the combination of two of the mechanically more relevant amino acid substitutions at each DNA-binding site.

The double-mutant D58A/N183A virion showed a reduced relative infectivity (about 0.01 ), but was still clearly infectious (Table 1). The purified D58A/N183A virion yielded $k_{\mathrm{e}}$ values similar to those obtained for the corresponding single mutants (Table 2). Thus, increasing the number of disrupted interactions does not necessarily lead to further reductions in mechanical stiffness. This non-additive effect may reflect, in mechanical terms, the well-established context-dependent (coupled) energetic effect of mutations of residues closely located within a ligand binding site in a protein.

\section{Reductions in local mechanical stiffness by removing capsid- DNA interactions are quantitatively linked to decreases in virion resistance against heat-induced inactivation}

When heated, MVM virions lose their infectivity in an irreversible, kinetically controlled process. Measurement of intrinsic fluorescence indirectly suggested that the virion is inactivated through a structural change that does not involve loss of overall physical integrity. ${ }^{47}$ We have now confirmed this suggestion. Purified virions were incubated at a high temperature for different amounts of time, and the percentages of infectious virions and of non-dissociated viral particles in the same experiment were respectively determined by titration and electron microscopy. As expected, infectivity was lost at a much faster rate than overall physical integrity (Fig. 4). By the time over $99 \%$ of the infectious virions had lost their infectivity, $50 \%$ of the viral particles were still undissociated. Both electron microscopy and AFM imaging revealed no differences between the infectious and inactivated virions. The mechanism of virion inactivation without losing capsid integrity remains to be determined. Among other possibilities, the viral DNA could have been extruded through the pores or a functional site in the capsid could have been structurally distorted.

We then addressed our tentative suggestion of a linkage in the MVM virion between mechanical stiffness at the regions that contain the DNA-bound sites (S2 and S3 regions) and resistance against the heat-induced structural transition that leads to inactivation of infectivity. We first obtained the inactivation rate constant for the non-mutated virion at $70{ }^{\circ} \mathrm{C}$ under defined conditions as described in the Experimental section. The experiments yielded $k_{\text {in }}=(5.4 \pm 1.6) \times 10^{-4} \mathrm{~s}^{-1}$ (Table 1).

We next subjected mutant virions D58A, N183A, W60A, N491A, T267A and D58A/N183A, and the non-mutated virion obtained in parallel (as an internal control included in each experiment), to heat-induced inactivation in physiological buffer. We had previously assessed the thermal behavior of some of these mutants semi-quantitatively, but the present

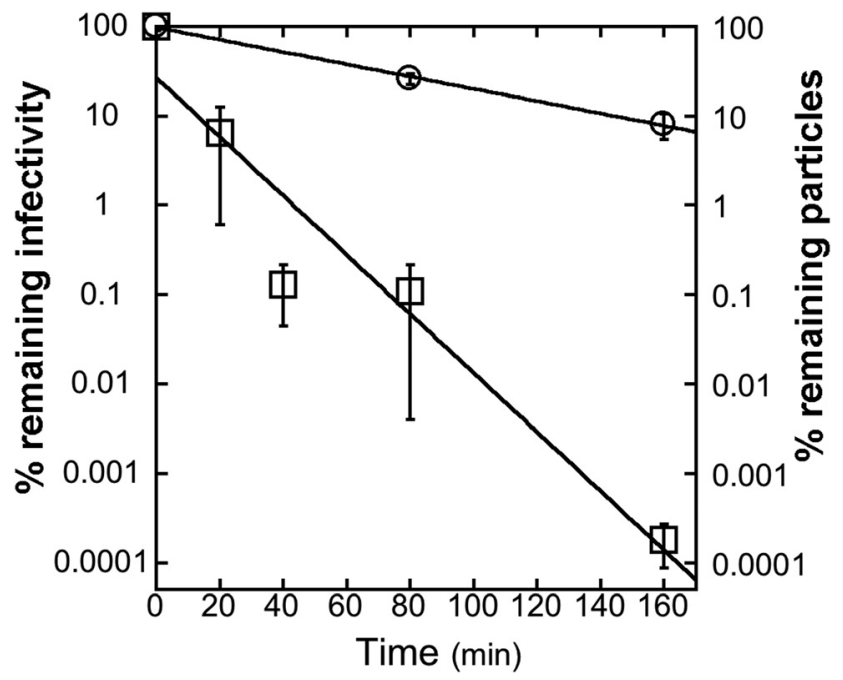

Fig. 4 Comparison of thermal inactivation kinetics and dissociation kinetics at $70{ }^{\circ} \mathrm{C}$. They were respectively determined by titration of residual virus infectivity and counting the remaining particles by electron microscopy for the same preparation of purified non-mutated MVM virion. Average and standard error values are represented in a semilogarithmic plot, and the data are fitted to exponential decay processes (lines).

experiments were required to include additional mutants to establish significant correlations, determine thermal sensitivity quantitatively, and compare the results with those of mechanical analysis of virus preparations obtained under the same conditions. Inactivation rate constants $k_{\text {in }}$ were obtained for each mutant and normalized relative to the $k_{\text {in }}$ obtained for the non-mutated virus in the same experiment. The relative $k_{\text {in }}$ values determined for each virus variant in different experiments were averaged (Table 1).

All six mutants showed a reduced resistance against thermal inactivation of their infectivity, yielding $k_{\text {in }}$ about 2-9-fold higher than that of the non-mutated virion (Fig. 5 and Table 1). Mutations D58A, N183A, W60A and D58A/N183A, which disrupted more energetic capsid-DNA interactions, led to large $k_{\text {in }}$ increases. Mutations N491A and T267A, which disrupted weaker capsid-DNA interactions, led to moderate $k_{\text {in }}$ increases.

A qualitative comparison between the $k_{\text {in }}$ and $k_{\mathrm{e}}$ values obtained for each virion (Tables 1 and 2) revealed a clear linkage in the MVM virion between decreased mechanical stiffness at each region around DNA-bound sites (S2 and S3 regions) and reduced resistance against thermal inactivation of infectivity. We tested next whether such a relationship could be quantitatively expressed by representing $k_{\text {in }}$ against $k_{\mathrm{e}}$ values for the six mutants and the non-mutated virion. As a measure of average stiffness for the region centered around each DNA-bound site, we used $k_{\mathrm{e}(\mathrm{s} 2 \mathrm{~s} 3)}$, the average of $k_{\mathrm{e}}$ values corresponding to $\mathrm{S} 2$ and $\mathrm{S} 3$ regions (Fig. 1C).

Remarkably, a simple exponential relationship was found between the rate constant for thermal inactivation of infectivity and the average mechanical stiffness at the MVM virion regions that include the DNA-binding sites (Fig. 6). The fitting 


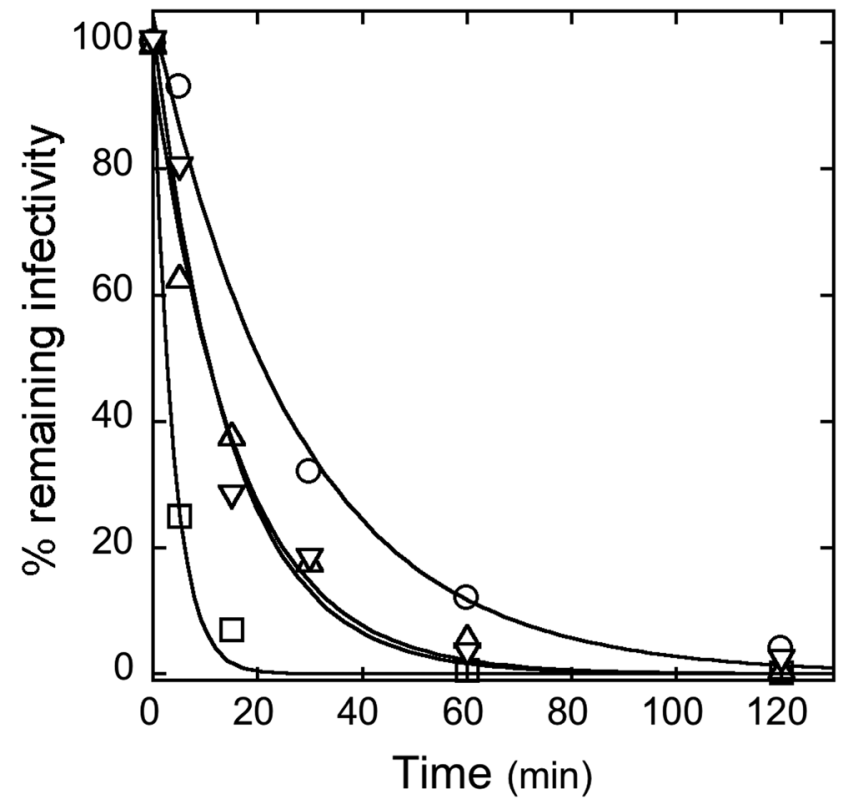

Fig. 5 Comparison of thermal inactivation kinetics at $70{ }^{\circ} \mathrm{C}$ between the non-mutated virion and some selected mutant MVM virions. Individual representative curves for selected virus variants are shown. For average and error values, and data for the complete series of mutants, see Table 1. Circles: non-mutated virion; squares: W60A; triangles: N491A; inverted triangles: T267A. Data were fitted to simple exponential processes (eqn (4)).

at the higher $k_{\mathrm{e}}$ values was slightly better than that shown in Fig. 6 if no restriction was imposed on the asymptote $k_{\text {in }}$ value. However, we imposed the restriction that at infinite rigidity $\left(k_{\mathrm{e}}=\infty\right)$ the inactivation reaction cannot occur $\left(k_{\mathrm{in}}=0\right)$, as justified in the Discussion section, where we provide a conceptual framework to explain this quantitative linkage.

Increases in mechanical stiffness by either establishing capsid-DNA interactions or filling cavities in the MVM capsid are non-additive

In $\mathrm{MVM}^{32}$ and other parvoviruses a conspicuous, poorly packed region leaving a cavity of about $300 \AA^{3}$ was found within the hydrophobic core of each of the 60 capsid subunits. This cavity is large enough to contain several water molecules. We had previously shown that amino acid substitutions that change the size and shape of these 60 equivalent cavities in the MVM capsid ${ }^{49}$ preserve the elasticity of the empty capsid at S5 regions, but increase by about two-fold its stiffness at S2 and S3 regions. ${ }^{46}$ This dramatic mechanical effect is comparable to that naturally achieved in the virion by binding DNA "wedges". ${ }^{36}$ As those cavities are located close to the DNA-binding sites (Fig. 1C), we decided to test whether the mechanical effects of DNA wedges and altered cavities could be combined to further increase the stiffness of the S2/S3 regions in the virion.

Mutant virions L113F and L143F in which small-to-large side chain replacements would partially fill and alter the shape of capsid cavities ${ }^{49}$ were produced, and were found to be as infectious as the parent virus (Table 1). Although both

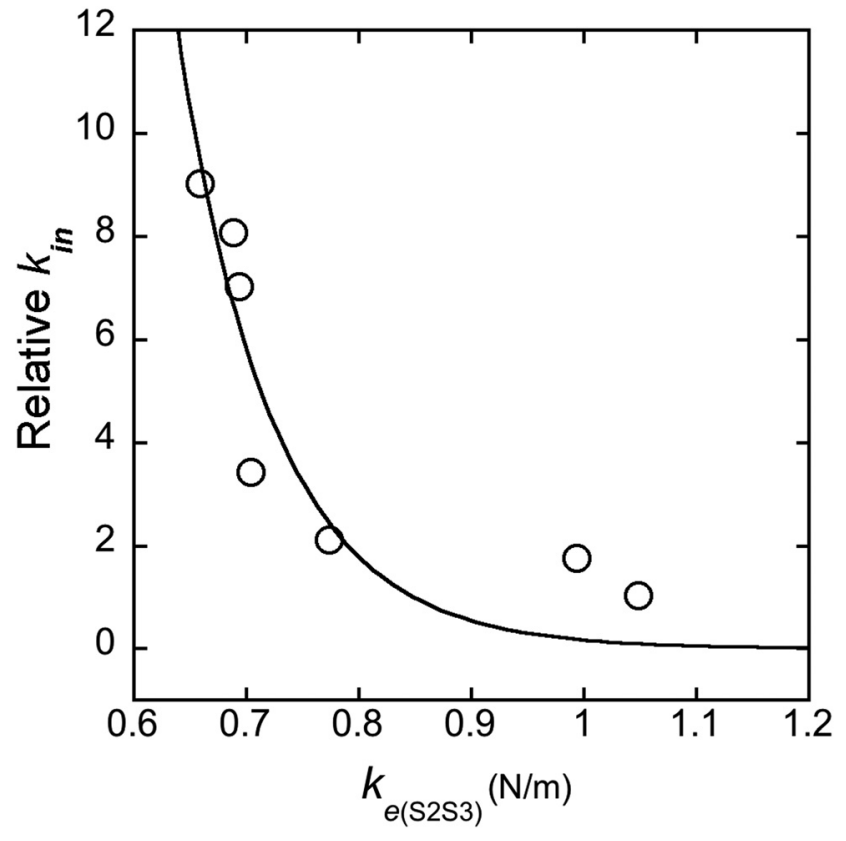

Fig. 6 Empirical relationship between thermal inactivation rate constant $\left(k_{\text {in }}\right)$ values and elastic constant values of S2/S3 regions $\left(k_{\text {e(s2s3) }}\right)$ for the non-mutated virion and different mutant virions with disrupted capsid-DNA interactions. Average $k_{\text {in }}$ and $k_{\mathrm{e}}$ values were fitted to a simple exponential process (eqn (3)). For statistical analysis see Tables 1 and 2 .

mutations increased stiffness at S2 and S3 regions in the empty capsid, ${ }^{46}$ they did not increase the stiffness of the same regions in the virion, already stiffened by the bound DNA segments (Fig. 3, S1 and S2† and Table 2). Such non-additive effects may arise from the relatively short distances between the capsid residues surrounding each cavity or involved in DNA binding (Fig. 1C).

The above result provided an additional opportunity to challenge the hypothesis of a linkage between mechanical stiffness at $\mathrm{S} 2 / \mathrm{S} 3$ regions in the MVM virion and resistance against heat-induced biological inactivation. As mutations L113F and L143F do not stiffen the virion, they were predicted not to increase its resistance against thermal inactivation either. Determination of the corresponding inactivation rate constants $k_{\text {in }}$ confirmed this prediction (Table 1).

\section{Reductions in local mechanical stiffness and decreased} resistance against thermal inactivation by removing capsidDNA interactions can be compensated by second-site substitutions that fill capsid cavities

Cavity-reshaping mutations like L113F and L143F did not increase S2/S3 stiffness in the virion beyond that achieved by the capsid-bound DNA wedges, but they did stiffen the DNAfree capsid. ${ }^{46}$ We reasoned that if stiffness of the S2/S3 regions in the virion were reduced to nearly that of the empty capsid by removing relevant capsid-DNA interactions, L113F or L143F substitutions could restore the original stiffness of the virion through a second-site compensatory effect. 
Double mutant virions D58A/L113F, D58A/L143F, N183A/ L113F and N183A/L143F with both disrupted capsid-DNA interactions and reshaped cavities were produced and titrated. Each single mutation had little or no effect on infectivity, but the double mutation was detrimental for virus infection. Mutant D58A/L113F was essentially lethal, but the remaining three mutants showed relative titers that were reduced by only 10-30-fold (Table 1) and could be purified.

These three double-mutant virions yielded $k_{\mathrm{e}}$ values at $\mathrm{S} 2 /$ S3 regions that are substantially higher than those obtained for the corresponding single mutants, approaching the values obtained for the non-mutated virion (Fig. 3, S1 and S2 $\uparrow$ and Table 2). Thus, reductions in mechanical stiffness caused by mutations that disrupt capsid-DNA interactions can be compensated by increases in stiffness caused by mutations that resize and reshape capsid cavities located nearby.

These results allowed us to challenge once more the hypothesis of a linkage in the MVM virion between increased mechanical stiffness at $\mathrm{S} 2 / \mathrm{S} 3$ regions and increased resistance against heat-induced inactivation. As mutant virions D58A/ L143F, N183A/L113F and N183A/L143F did recover stiffness at $\mathrm{S} 2 / \mathrm{S} 3$ regions, lost by removing capsid-DNA interactions, they should also recover resistance against thermal inactivation. Determination of the inactivation rate constants $k_{\text {in }}$ for the three cavity/DNA site double mutants revealed that, although not completely restored, resistance against heatinduced inactivation was substantially increased compared to that of the single mutants D58A and N183A, as predicted (Table 1).

\section{Discussion}

The hypothesis of a relationship between increased mechanical stiffness in a region of a virus particle, and decreased propensity for a structural change in which that region is involved, is now supported for two independent processes in MVM: (i) increased stiffness of the capsid S5 regions by mutations at the base of the pores is inextricably linked to the impairment of a pore-associated conformational rearrangement required for virus infection; ${ }^{46}$ and (ii) decreased stiffness of the $\mathrm{S} 2 / \mathrm{S} 3$ regions by mutations that disrupt capsid-DNA interactions in those regions is invariably associated with an increased propensity for a heat-induced structural transition that leads to virus inactivation (this study).

The present work has also revealed that the association between decreased propensity for a structural rearrangement and increased stiffness follows an empirical exponential relationship. This relationship can be justified based on transition state theory, by making the assumption (discussed below) that there is a direct linear relationship between the degree of mechanical stiffness of a particular region in a viral particle, and the height of the free energy barrier $\Delta G^{\ddagger}$ of a structural transition in which that region is involved:

$$
\Delta G^{\ddagger}=C k_{\mathrm{e}}
$$

where $C$ is an empirical factor. The value of $C$ would depend on the virus, region, and conformational transition being considered.

According to the Eyring equation:

$$
k_{\text {in }}=\left(K k_{\mathrm{B}} T / h\right) \mathrm{e}^{\left(-\Delta G^{\ddagger} / k_{\mathrm{B}} T\right)}
$$

where $k_{\text {in }}$ is the rate constant of the reaction; $\left(K k_{\mathrm{B}} T / h\right)$ is a preexponential factor (reaction-specific quantity) that corresponds to the maximum rate constant of the reaction; $K$ is the transmission coefficient, $k_{\mathrm{B}}$ is the Boltzmann constant, $T$ is the absolute temperature and $h$ is the Planck constant; $\Delta G^{\ddagger}$ is the free energy barrier (Gibbs energy of activation) of the reaction, i.e., the difference between the free energy of the ground state and that of the transition state $(\vdots)$.

By substituting $\Delta G^{\ddagger}=C k_{\mathrm{e}}$ (1) in the Eyring eqn (2):

$$
k_{\text {in }}=\left(K k_{\mathrm{B}} T / h\right) \mathrm{e}^{\left(-C k_{\mathrm{e}} / k_{\mathrm{B}} T\right)}
$$

which describes an exponential relationship between $k_{\text {in }}$ and $k_{\mathrm{e}}$, as experimentally found (Fig. 6).

From the best fitting of data to eqn (3), $C=5.55 \times 10^{-20} \mathrm{~m}^{2}$. Thus, for each increase in mechanical stiffness $\Delta k_{\mathrm{e}}=0.1 \mathrm{~N}$ $\mathrm{m}^{-1}$ at the $\mathrm{S} 2 / \mathrm{S} 3$ regions, the free energy barrier for inactivation of MVM increases by $\Delta \Delta G^{*}=5.55 \times 10^{-21} \mathrm{~J}$ per virus particle $\left(3.34 \mathrm{~kJ} \mathrm{~mol}^{-1}\right)$ at $70{ }^{\circ} \mathrm{C}$ under the conditions used. Extrapolation for $k_{\mathrm{e}}=0.6 \mathrm{~N} \mathrm{~m}^{-1}$, the stiffness of the $\mathrm{S} 2 / \mathrm{S} 3$ regions in the natural DNA-free capsid, ${ }^{35}$ yields a $k_{\text {in }}$ about 19 -fold higher than the $k_{\text {in }}$ for the natural MVM virion. Thus, the increased mechanical stiffness of the $\mathrm{S} 2 / \mathrm{S} 3$ regions in MVM, achieved by biologically evolving ssDNA-binding sites in the capsid, can be translated into: (i) a $35 \mathrm{~kJ} \mathrm{~mol}^{-1}$ increase in the free energy barrier of the virus inactivation reaction; and (ii) a 19-fold increased half-life of the infectious virion (from about $67 \mathrm{~s}$ to $21 \mathrm{~min})$ when subjected to thermal stress $\left(70{ }^{\circ} \mathrm{C}\right.$ under the conditions used).

Why a linear relationship exists between mechanical stiffness of a region in a viral particle and the free energy barrier $\Delta G^{\ddagger}$ of a structural transition in which that region is involved is discussed next. Structural transitions in protein molecules or complexes, such as ligand unbinding, polypeptide unfolding, conformational rearrangements or subunit release, can be mechanically induced by applying force in single-molecule experiments by "pulling" or "pushing". ${ }^{50-59}$ In the simplest case (a two-state reaction), $\Delta G^{\ddagger}$ is overcome when enough mechanical force (helped by thermal force) is applied to stretch the molecule by a length that corresponds to the position of the transition state along the mechanical reaction coordinate. The observed non-linear molecular deformation reflects large changes in the relative positions of many atoms, achieved through disruption of non-covalent interactions and/or a reduction of the hydrophobic effect.

When "pushing" experiments are instead performed to determine virus stiffness, particles are likewise deformed, but in an elastic, reversible process. ${ }^{15}$ Multiple non-covalent interactions between atoms are stressed or (gradually) disrupted, 
and atoms will still be displaced, albeit not enough for reaching their positions in the transition state and triggering any structural transition the particle may be prone to.

Let us consider now a structural modification in a virus particle that increases the overall strength of the interactions holding the atoms within a particular region close to their equilibrium positions. If this region is involved in a conformational transition, $\Delta G^{\ddagger}$ could be expected to increase. From basic physics (work $=$ force $\times$ distance), it could be said that more force will be required to move atoms from their equilibrium positions in the native state to their equilibrium positions in the transition state of the reaction. Likewise, it could be then expected that more mechanical force $(F)$ will have to be applied by indentation to achieve a certain deformation (compression length, $d$ ). Given that $k_{\mathrm{e}}=F / d$, the elastic constant could be expected to increase in proportion to the increase in $\Delta G^{\ddagger}$, as observed.

The direct linear relationship found between $k_{\mathrm{e}}$ and $\Delta G^{\ddagger}$ is also satisfying for extreme values of $k_{\mathrm{e}}$ : (i) if the virus particle could not be deformed even by the application of infinite mechanical force $\left(k_{\mathrm{e}}=\infty\right)$, atoms could not be displaced at all, and $\Delta G^{\ddagger}=\infty$; (ii) if atoms involved in the structural change could freely move even when no mechanical force is applied $\left(k_{\mathrm{e}}=0\right)$, then $\Delta G^{\ddagger}=0$ and the reaction rate would reach its maximum (eqn (3)).

It must be emphasized that what we found is a linkage between variations in stiffness and activation energy in a same particle region, and not between absolute values. The value of $C$ may depend on the particular atomic groups, interactions, and conformational transition considered. It must also be remarked that the intrinsic elasticity of a solid material is measured by the Young's modulus $E$ and not by $k_{\mathrm{e}}$ which depends not only on the material, but also on geometric considerations. However, as we are considering a same region of a same virus, the geometry is preserved and $k_{\mathrm{e}}$ can be directly used as a measure of the relative intrinsic stiffness of the material and is directly related to $\Delta G^{\ddagger}$.

When different structural modifications were made in one capsid element only (the DNA-binding sites), the exponential relationship between $k_{\mathrm{e}}$ and $k_{\text {in }}$ was quite good. However, when stiffness was varied by more complex, compensatory modifications involving DNA-binding sites and cavities, the relationship still held, but deviated from ideality. Some possible causes can be advanced.

(i) If deformability of a virus region is anisotropic, and such anisotropy is modified by a structural change, the variation in $k_{\mathrm{e}}$ measured along one direction only could fail to provide a good estimation of the overall change in deformability. Anisotropic deformability may indeed be frequent in protein domains, either as a side effect of topology or by natural selection (in elastomeric proteins with $\beta$-sheet "mechanical clamps"). ${ }^{60,61}$ However, in the large regions of a virus capsid that are deformed by an AFM tip, a strong alignment of directional interactions could be unusual, limiting the dependency of $k_{\mathrm{e}}$ on pushing geometry and preserving the correlation between $k_{\mathrm{e}}$ and $\Delta G^{\ddagger}$. (ii) The subsets of atoms that may change relative positions by either mechanical deformation or in a structural transition may not be necessarily identical. However, those subsets may overlap considerably if a large region of a viral particle is involved, again limiting deviations from the ideal relationship between $k_{\mathrm{e}}$ and $\Delta G^{\ddagger}$.

The relationship between local mechanical elasticity and propensity for structural transitions found for MVM may not depend conceptually on the structural complexity of the viral particle. Thus, it may hold true for larger viruses, unless obscured by complications such as those considered in points (i) or (ii) above. This aspect remains to be investigated.

From a virological perspective, this study provides further support and expands our hypothesis on the biological relevance of the anisotropic distribution of mechanical elasticity in the MVM virion ${ }^{46}$ (see Fig. 1C). MVM appears to have evolved, by positive selection, DNA-binding sites in S2/S3 regions at the capsid inner wall, which provide the biological advantage of an increased resistance against thermal inactivation. The DNA-bound wedges increase the mechanical stiffness of the S2/S3 regions and impair a heat-induced structural transition leading to loss of infectivity. In contrast, negative selection may have kept the S5 regions around the capsid pores free of bound DNA, and mechanically elastic enough to facilitate a different conformational transition, which is associated with translocation events through the pores and is biologically required for viral infection.

In modified virions where some capsid-DNA interactions had been removed, single mutations that resized and reshaped capsid cavities led also to increased stiffness at S2/S3 regions and resistance against thermal inactivation. Thus, the virus could have evolved thermal resistance by reshaping cavities instead of creating DNA-binding sites. However, it "chose" to do the latter, perhaps because cavity size and shape need to be preserved for viral function. This possibility is supported by the fairly conserved size of these cavities in related parvoviruses and the finding that some changes in cavity size/shape reduce virus infectivity. ${ }^{33,48,49}$ It appears that partly contradictory selective pressures have exquisitely tuned the anisotropic mechanical stiffness of this simple virus by natural "engineering" of capsid architectural elements, including DNA-binding sites, cavities, and the annular regions at the base of the pores.

From a nanotechnological perspective, this study provides proof of principle that engineered changes in the propensity of protein-based assemblies for undergoing structural rearrangements can be readily and quantitatively probed by determining mechanical stiffness in single-molecule indentation experiments using AFM. Such an analysis may be most helpful in the development of modified virus-based and other proteinbased nanoparticles that have (i) the correct propensity for structural changes required for their intended application (e.g., release of the cargo for targeted drug delivery); or (ii) enough structural resilience against detrimental conformational rearrangements (e.g., those affecting the functional structure and/or relative position of active moieties in nanosensors or nanostructured materials). 


\section{Experimental}

\section{Recombinant plasmids and mutagenesis}

The QuikChange Site-directed mutagenesis kit (Stratagene) was used to introduce specific mutations on recombinant plasmid pSVtk-VP1/2, ${ }^{62}$ which contains the capsid proteincoding gene of MVM strain p (MVMp). The same mutations were introduced into an MVMp infectious clone ${ }^{63}$ by subcloning the relevant segment from the corresponding mutant plasmid pSVtk-VP1/2. ${ }^{49,64}$ The presence of the introduced mutations and the absence of further mutations were confirmed by automated DNA sequencing.

\section{Purification of MVM virions}

DNA-filled MVM virions were obtained from NB324K cells transfected by electroporation with non-mutated and mutant MVM infectious plasmids and purified as previously described. ${ }^{45,64}$ Purified virions were quantified in hemagglutination assays ${ }^{65}$ and stored at $4{ }^{\circ} \mathrm{C}$ until use.

\section{Infectivity assays}

NB324K cells were transfected by electroporation using equal amounts of mutant and non-mutated infectious plasmids and the non-mutated control. Protein expression was quantified in Western blot assays. ${ }^{45}$ Infectious progeny viruses were quantified by titration in standard plaque-formation assays. To ensure comparable results, a control non-mutated virus was included in each experiment. Titers were obtained in duplicate, averaged and expressed as relative values with respect to the titer obtained in parallel for the non-mutated virus in the same experiment. Finally, the mean relative titers obtained for each mutant in two or three independent experiments were averaged.

\section{Thermal inactivation assays}

Virions obtained 96 hours after transfection of NB324K cells with comparable high titers (around $10^{5}$ plaque-forming units $\left.\mathrm{ml}^{-1}\right)$ in physiological medium $(1 \mathrm{ml})$ were incubated at $70{ }^{\circ} \mathrm{C}$ for different amounts of time. Infectious virions remaining in each case were titrated in duplicate. Inactivation rate constants $k_{\text {in }}$ were obtained by fitting the experimental data to first-order exponential decays with no remaining infectivity at infinite time, using the program Kaleidagraph (Synergy Software), and the equation:

$$
I=I_{0} \mathrm{e}^{\left(-k_{\text {in }} t\right)}
$$

where $I$ is the infectivity titer at time $t, I_{0}$ is the infectivity titer at $t=0$, and $k_{\text {in }}$ is the inactivation rate constant. For each mutant, the $k_{\text {in }}$ was normalized relative to that determined in the same experiment for a non-mutated control virus obtained in a parallel transfection. Finally, the relative $k_{\text {in }}$ values obtained for each mutant in two or three independent experiments were averaged.

To directly estimate the relative amount of non-dissociated virus particles after different incubation times at $70{ }^{\circ} \mathrm{C}$, ali- quots were taken and virus particles in each aliquot were visualized by transmission electron microscopy using negative staining and counted.

\section{Imaging virus particles by AFM}

AFM experiments were carried out essentially as described earlier. ${ }^{35,36}$ The atomic force microscope (Nanotec Electrónica S.A.) was operated in jumping mode ${ }^{66}$ in a liquid. RC800PSA cantilevers (Olympus) with a nominal elastic constant of $k_{\mathrm{c}}=$ $0.1 \mathrm{~N} \mathrm{~m}^{-1}$ were used. Purified MVM particles were adsorbed on silanized glass and kept in PBS during imaging. The maximum normal force was about 100 pN. AFM images were processed by using WSxM software. ${ }^{67}$

\section{Determination of mechanical stiffness by AFM}

Mechanical stiffness of different regions of MVM particles was determined by AFM as previously described. ${ }^{3,36}$ RC800PSA cantilevers (Olympus) with a nominal elastic constant of $k_{\mathrm{c}}=$ $0.1 \mathrm{~N} \mathrm{~m}^{-1}$ were used. The actual $k_{\mathrm{c}}$ of each cantilever used was determined as previously described. ${ }^{68}$ To keep the system within the range of a linear elastic response and avoid particle damage, only force-versus-distance (Fz) measurements that involved indentations between $0.5 \mathrm{~nm}$ and $2.0 \mathrm{~nm}$ were considered. If after a contact event the $\mathrm{Fz}$ curve exhibited a marked step (by irreversible modification of the particle) we moved to another particle. The elastic constant of the particle at the region indented along the direction of the applied force, $k_{\mathrm{e}}$, was obtained from the experimentally determined elastic constant value by assuming that virus particle and cantilever behave as two ideal springs in series. ${ }^{15}$

\section{Statistical analysis of elastic constant values}

The $k_{\mathrm{e}}$ values followed a normal distribution described by a Gaussian fitting, as corroborated using normality tests, and observed for other virus particles. The statistical significance of $k_{\mathrm{e}}$ differences was evaluated using OriginPro8 (OriginLab). To statistically validate that two $k_{\mathrm{e}}$ distributions are different, the two-population two-tailed Student's $t$-test was used, with an alpha level $=0.05$. Equal variance was not assumed, providing a more stringent test.

\section{Molecular graphics and structural analyses}

The programs InsightII (Biosym Technologies), Whatif, ${ }^{69}$ Chimera, ${ }^{70} \mathrm{VMD}^{71}$ and the PDB atomic coordinates for the crystal structures of MVM virion (1Z1C) and empty capsid $(1 \mathrm{Z14})^{32,33}$ were used to analyze structures, design mutations, interpret results and/or graphic display.

\section{Conclusion}

The experimental evidence obtained with MVM quantitatively supports the following hypothesis: in a viral particle, any structural change that decreases the mechanical stiffness of a region involved in a conformational transition may facilitate such a transition by reducing the free energy barrier of the 
reaction; conversely, any structural change that increases stiffness will impair the transition. From the reciprocal point of view, any structural change in a viral particle that either facilitates or impairs a conformational transition may respectively decrease or increase the mechanical stiffness of the viral region involved. From a virological perspective, the results provide strong evidence for the biological relevance of the complex distribution of mechanical stiffness in an infectious virion. From a nanotechnological perspective, the results provide proof of principle that relative local elasticity, as determined by AFM, may provide a simple mechanical sensor of variations in propensity for specific structural transitions in viruses, viral nanoparticles and other protein-based nanocomplexes that have been chemically or genetically modified. By applying eqn (3), the variation in activation free energy and rate constant of the reaction can be predicted from the variation in mechanical stiffness.

\section{Acknowledgements}

We thank A. Valbuena for reading the manuscript, A. Valbuena, P.J. de Pablo and J. Gómez-Herrero for fruitful discussions, expert help and support with AFM analysis, J.M. Almendral for generous collaboration in MVM studies, M. Alcorlo for technical help with Fig. 1, and A. RodríguezHuete and M.A. Fuertes for excellent technical assistance. This work was funded by grants to M.G.M. from the Spanish Government (BIO2009-10092 and BIO2012-37649) and Comunidad de Madrid (S-505/MAT-0303) and by an institutional grant from Fundación Ramón Areces to the Centro de Biología Molecular. P.J.P.C. is the recipient of a FPI contract from the Spanish Government. M.G.M. is an associate member of the Institute for Biocomputation and Physics of Complex Systems, Zaragoza, Spain.

\section{References}

1 T. Douglas and M. Young, Science, 2006, 312, 873-875.

2 K. T. Kim, S. A. Meeuwissen, R. J. M. Nolte and J. C. M. van Hest, Nanoscale, 2010, 2, 844-858.

3 M. G. Mateu, Protein Eng., Des. Sel., 2011, 24, 53-63.

4 A. M. Bittner, J. M. Alonso, M. L. Górzny and C. Wege, in Structure and physics of viruses, ed. M. G. Mateu, Springer, Dordrecht, The Netherlands, 2013, ch. 22, pp. 667-702.

5 A. M. Wen, P. H. Rambhia, R. H. French and N. F. Steinmetz, J. Biol. Phys., 2013, 39, 301-325.

6 J. Glasgow and D. Tullman-Ercek, Appl. Microbiol. Biotechnol., 2014, 98, 5847-5858.

7 F. Li and Q. Wang, Small, 2014, 10, 230-245.

8 C. M. Knobler and W. M. Gelbart, Annu. Rev. Phys. Chem., 2009, 60, 367-383.

9 W. H. Roos, R. Bruinsma and G. J. L. Wuite, Nat. Phys., 2010, 6, 733-743.
10 Emerging Topics in Physical Virology, ed. P. G. Stockley and R. Twarock, Imperial College Press, London, UK, 2010.

11 Structure and Physics of Viruses, ed. M. G. Mateu, Springer, Dordrecht, The Netherlands, 2013.

12 I. L. Ivanovska, P. J. de Pablo, B. Ibarra, G. Sgalari, F. C. MacKintosh, J. L. Carrascosa, C. F. Schmidt and G. J. L. Wuite, Proc. Natl. Acad. Sci. U. S. A., 2004, 101, 7600-7605.

13 W. H. Roos and G. J. L. Wuite, Adv. Mater., 2009, 21, 11871192.

14 M. G. Mateu, Virus Res., 2012, 168, 1-22.

15 P. J. de Pablo and M. G. Mateu, in Structure and physics of viruses, ed. M. G. Mateu, Springer, Dordrecht, The Netherlands, 2013, ch. 18, pp. 519-551.

16 M. Hernando-Pérez, S. Lambert, E. Nakatani-Webster, C. E. Catalano and P. J. de Pablo, Nat. Commun., 2014, 5, 4520.

17 M. Hernando-Pérez, E. Pascual, M. Aznar, A. Ionel, J. R. Castón, A. Luque, J. L. Carrascosa, D. Reguera and P. J. de Pablo, Nanoscale, 2013, 6, 2702-2709.

18 P. J. de Pablo, in Structure and physics of viruses, ed. M. G. Mateu, Springer, Dordrecht, The Netherlands, 2013, ch. 8, pp. 247-271.

19 A. Luque and D. Reguera, in Structure and physics of viruses, ed. M. G. Mateu, Springer, Dordrecht, The Netherlands, 2013, ch. 19, pp. 553-595.

20 H. B. Pang, L. Hevroni, N. Kol, D. M. Eckert, M. Tsvitov, M. S. Kay and I. Rousso, Retrovirology, 2013, 10, 4.

21 J. M. Hogle, Annu. Rev. Microbiol., 2002, 56, 677-702.

22 J. E. Johnson, Adv. Protein Chem., 2003, 64, 197-218.

23 A. C. Steven, J. B. Heymann, N. Cheng, B. L. Trus and J. F. Conway, Curr. Opin. Struct. Biol., 2005, 15, 227-236.

24 T. J. Tuthill, E. Groppelli, J. M. Hogle and D. J. Rowlands, Curr. Top. Microbiol. Immunol., 2010, 343, 43-89.

25 B. Bothner and J. K. Hilmer, in Structural Virology, ed. M. Agbandje-McKenna and R. McKenna, RSC Publishing, Cambridge, UK, 2011, ch. 3, pp. 41-61.

26 D. Veesler and J. E. Johnson, Annu. Rev. Biophys., 2012, 41, 473-496.

27 M. Suomalainen and U. F. Greber, Curr. Opin. Virol., 2013, 3, 27-33.

28 M. G. Mateu, Arch. Biochem. Biophys., 2013, 532, 65-79.

29 A. T. Da Poian, J. E. Johnson and J. L. Silva, J. Biol. Chem., 2002, 277, 47596-47602.

30 R. Mateo, E. Luna, V. Rincón and M. G. Mateu, J. Virol., 2008, 82, 12232-12240.

31 J. M. Sanchez-Ruiz, Biophys. Chem., 2010, 148, 1-15.

32 M. Agbandje-McKenna, A. L. Llamas-Saiz, F. Wang, P. Tattersall and M. G. Rossmann, Structure, 1998, 6, 13691381.

33 M. Kontou, L. Govindasamy, H. J. Nam, N. Bryant, A. L. Llamas-Saiz, C. Foces-Foces, E. Hernando, M. P. Rubio, R. McKenna, J. M. Almendral and M. Agbandje-McKenna, J. Virol., 2005, 79, 10931-10943.

34 P. Plevka, S. Hafenstein, L. Li, A. D’Abramo Jr., S. F. Cotmore, M. G. Rossmann and P. Tattersall, J. Virol., 2011, 85, 4822-4827. 
35 C. Carrasco, A. Carreira, I. Schaap, P. Serena, J. GómezHerrero, M. G. Mateu and P. J. de Pablo, Proc. Natl. Acad. Sci. U. S. A., 2006, 103, 13706-13711.

36 C. Carrasco, M. Castellanos, P. J. de Pablo and M. G. Mateu, Proc. Natl. Acad. Sci. U. S. A., 2008, 105, 4150-4155.

37 A. Carreira, M. Menéndez, J. Reguera, J. M. Almendral and M. G. Mateu, J. Biol. Chem., 2004, 279, 6517-6525.

38 J. Reguera, A. Carreira, L. Riolobos, J. M. Almendral and M. G. Mateu, Proc. Natl. Acad. Sci. U. S. A., 2004, 101, 27242729.

39 S. F. Cotmore, A. M. D’Abramo Jr., C. M. Ticknor and P. Tattersall, Virology, 1999, 254, 169-181.

40 E. Hernando, A. L. Llamas-Saiz, C. Foces-Foces, R. McKenna, I. Portman, M. Agbandje-McKenna and J. M. Almendral, Virology, 2000, 267, 299-309.

41 B. Maroto, N. Valle, R. Saffrich and J. M. Almendral, J. Virol., 2004, 78, 10685-10694.

42 N. Valle, L. Riolobos and J. M. Almendral, in Parvoviruses, ed. J. R. Kerr, S. F. Cotmore, M. E. Bloom, R. M. Linden and C. R. Parrish, Edward Arnold, London, UK, 2006, ch. 20, pp. 291-304.

43 S. F. Cotmore and P. Tattersall, Adv. Virus Res., 2007, 70, 183-232.

44 S. F. Cotmore and P. Tattersall, J. Virol., 2012, 86, 69-80.

45 C. Sánchez-Martínez, E. Grueso, M. Carroll, J. Rommelaere and J. M. Almendral, Virology, 2012, 432, 45-56.

46 M. Castellanos, R. Pérez, C. Carrasco, M. Hernando-Pérez, J. Gómez-Herrero, P. J. de Pablo and M. G. Mateu, Proc. Natl. Acad. Sci. U. S. A., 2012, 109, 12028-12033.

47 J. Reguera, E. Grueso, A. Carreira, C. Sánchez-Martínez, J. M. Almendral and M. G. Mateu, J. Biol. Chem., 2005, 280, 17969-17977.

48 M. S. Chapman and M. G. Rossmann, Structure, 1995, 3, 151-162.

49 A. Carreira and M. G. Mateu, J. Mol. Biol., 2006, 360, 10811093.

50 T. E. Fisher, P. E. Marszalek and J. M. Fernandez, Nat. Struct. Biol., 2000, 7, 719-724.

51 C. Bustamante, Y. R. Chemla, N. R. Forde and D. Izhaky, Annu. Rev. Biochem., 2004, 73, 705-748.

52 F. Kienberger, A. Ebner, H. J. Gruber and P. Hinterdorfer, Acc. Chem. Res., 2006, 39, 29-36.

53 A. Borgia, P. M. Williams and J. Clarke, Annu. Rev. Biochem., 2008, 77, 101-125.
54 A. F. Oberhauser and M. Carrión-Vázquez, J. Biol. Chem., 2008, 283, 6617-6621.

55 C. Seifert and F. Gräter, Biochim. Biophys. Acta, 2013, 1839, 4762-4768.

56 B. Jagannathan and S. Marqusee, Biopolymers, 2013, 99, 860-869.

57 I. L. Ivanovska, R. Miranda, J. L. Carrascosa, G. J. Wuite and C. F. Schmidt, Proc. Natl. Acad. Sci. U. S. A., 2011, 108, 12611-12616.

58 M. Castellanos, R. Pérez, P. J. P. Carrillo, P. J. de Pablo and M. G. Mateu, Biophys. J., 2012, 102, 2615-2624.

59 A. J. Pérez-Berná, A. Ortega-Esteban, R. Menéndez-Conejero, D. C. Winkler, M. Menéndez, A. C. Steven, S. J. Flint, P. J. de Pablo and C. San Martín, J. Biol. Chem., 2012, 287, 31582-31595.

60 D. J. Brockwell, E. Paci, R. C. Zinober, G. S. Beddard, P. D. Olmsted, D. A. Smith, R. N. Perham and S. E. Radford, Nat. Struct. Biol., 2003, 10, 731-737.

61 M. Carrión-Vázquez, H. Li, H. Lu, P. E. Marszalek, A. F. Oberhauser and J. M. Fernandez, Nat. Struct. Biol., 2003, 10, 738-743.

62 J. C. Ramirez, J. F. Santarén and J. M. Almendral, Virology, 1995, 206, 57-68.

63 E. M. Gardiner and P. Tattersall, J. Virol., 1998, 62, 26052613.

64 E. Lombardo, J. C. Ramírez, J. García and J. M. Almendral, J. Virol., 2002, 76, 7049-7059.

65 M. P. Rubio, A. López-Bueno and J. M. Almendral, J. Virol., 2005, 79, 11280-11290.

66 F. Moreno-Herrero, P. J. de Pablo, R. Fernández-Sánchez, J. Colchero, J. Gómez-Herrero and A. Baró, Appl. Phys. Lett., 2002, 81, 2620-2622.

67 I. Horcas, R. Fernández, J. M. Gómez-Rodríguez, J. Colchero, J. Gómez-Herrero and A. M. Baró, Rev. Sci. Instrum., 2007, 78, 013705.

68 J. E. Sader, J. W. M. Chon and P. Mulvaney, Rev. Sci. Instrum., 1999, 70, 3967-3969.

69 G. Vriend, J. Mol. Graphics, 1990, 8, 52-56.

70 E. F. Pettersen, T. D. Goddard, C. C. Huang, G. S. Couch, D. M. Greenblatt, E. C. Meng and T. E. Ferrin, J. Comput. Chem., 2004, 25, 1605-1612.

71 W. Humphrey, A. Dalke and K. Schulten, J. Mol. Graphics, 1996, 14, 33-38. 